\title{
The Effect of Hyperoxia on Reactive Oxygen Species (ROS) in Rat Petrosal Ganglion Neurons During Development Using Organotypic Slices
}

\author{
DANIEL J. KWAK, SPENCER D. KWAK, AND ESTELLE B. GAUDA
}

Johns Hopkins University School of Medicine, Baltimore, Maryland, 21287

\begin{abstract}
Hyperoxia, during development in rats, results in hypoxic chemosensitivity ablation, carotid body hypoplasia, and reduced chemoafferents. We hypothesized that hyperoxia increases reactive oxygen species (ROS) in cell bodies of chemoafferents. Organotypic slices of petrosal-nodose ganglia from rats at day of life (DOL) 5-6 and 17-18 were exposed to $8 \%, 21 \%$, or $95 \% \mathrm{O}_{2}$ for $4 \mathrm{~h}$ in the presence or absence of the ROS-sensitive fluorescent indicator, $\mathrm{CM}-\mathrm{H}_{2}$ DCFDA, and propidium iodide was used to determine the relationship between cell death and oxygen tension. In tissue slices from DOL 5-6 rats, fluorescence intensity was $182.5 \pm 2.9$ for hypoxia, $217.5 \pm 3.3$ for normoxia, and $336.6 \pm 3.8$ for hyperoxia, (mean \pm SEM, $p<0.001$, ANOVA). Normoxia increased ROS levels by $19.2 \%$ from hypoxia $(p<0.01)$ with a further increase of $54.8 \%$ from normoxia to hyperoxia $(p<0.001)$. In tissue slices from DOL 17-18 rats, ROS levels increased with increasing oxygen tension but were less than in younger animals ( $p<0.01$, ANOVA). The antioxidants, NAC and TEMPO-9-AC, attenuated ROS levels and cell death. Electron microscopy demonstrated that hyperoxia damages the ultrastructure within petrosal ganglion neurons. Hyperoxic-induced increased levels of ROS in petrosal ganglion neurons may contribute to loss of hypoxic chemosensitivity during early postnatal development. (Pediatr Res 60: 371-376, 2006)
\end{abstract}

$\mathrm{N}^{\prime \prime m}$ umerous studies in mammalian species support a role for peripheral arterial chemoreceptors in stabilizing ventilation at a critical period during early postnatal development, which establishes rhythmogenesis that is sustained throughout life $(1,2)$. The components of the peripheral arterial chemoreceptors are found within the carotid body that is located in the bifurcation of the carotid artery and consists of three major neuronal components that include: 1) type I chemosensory cells, also known as glomus cells, which contain neurotransmitters and autoreceptors; 2) type II cells, which are similar to supportive glial cells; and 3) chemoafferent nerve fibers from the carotid sinus nerve, a branch of the IX cranial nerve, with cell bodies in the petrosal ganglion (PG) $(3,4)$.

Received January 11, 2006; accepted June 4, 2006

Correspondence: Estelle B. Gauda, M.D., The John Hopkins Hospital, Division of Neonatology, Research Laboratories, 600 North Wolfe Street, CMSC 6-104, Baltimore, MD, 21287-3200; e-mail: egauda@.jhmi.edu

This investigation was supported by grants from the National Institute on Drug Abuse (DA 013940-03) and the National Institutes of Health (HL 072748).

DOI: 10.1203/01.pdr.0000239817.39407.61
Exposure to chronic hyperoxia, during the first weeks of postnatal development, depresses ventilatory responses to subsequent acute hypoxia in newborn animals and in premature infants (5-7). Hyperoxic exposure in newborn rat pups is cytotoxic to peripheral arterial chemoreceptors as evidenced by hypoplasia of the carotid body and a $41 \%$ reduction in the number of chemoafferent neurons (8). The mechanisms leading to cytotoxic changes in the carotid body and the reduction in chemoreflexes after hyperoxicexposure during early postnatal development are unknown. In other model systems, hyperoxia is associated with increased production of ROS, including superoxide, hydroxyl radical, and hydrogen peroxide, which can contribute to cellular damage via lipid peroxidation, enzyme inactivation, and protein and nucleic acid oxidation, resulting in apoptosis or necrosis (9). Using a novel ex vivo organotypic slice culture system, we hypothesize that hyperoxia leads to an increased level of ROS and subsequent cytotoxic response in cell bodies of the chemoafferent neurons located in the $\mathrm{PG} / \mathrm{NG}$, and this response is augmented during early postnatal development.

\section{METHODS}

Rat pups born to time-dated Sprague-Dawley dams were used in experimental protocols approved by the Animal Care and Use Committee at Johns Hopkins University. The rat pups at postnatal day of life (DOL) 5-6 and 17-18 ( $n=6$ at each age) were briefly anesthetized with halothane and rapidly decapitated. To control for litter affects, a maximum of two rat pups were used per litter in each experimental group. The carotid body and PG/NG complex were rapidly removed en bloc and placed in ice-cold Leibovitz's L-15 media (Invitrogen, Carlsbad, CA). The tissue complex was then embedded in 3\% low melting point agar (Sigma Chemical Co., St. Louis, MO). Embedded tissues bathed in Leibovitz's L-15 media were sectioned at $45 \mu \mathrm{m}$ with a vibrating Vibratome (Ted Pella, Inc., Redding, CA), with a blade angle of $35^{\circ}$, a speed of 2 , and an amplitude of 8.5 , at $1{ }^{\circ} \mathrm{C}$, and then placed in Neurobasal media (Invitrogen) supplemented with $0.3 \%$ L-glutamine (Invitrogen) and 2\% B-27 with antioxidants (Invitrogen) and 1\% Pen-Strept (Invitrogen) and incubated at $37^{\circ} \mathrm{C}\left(21 \% \mathrm{O}_{2} / 5 \% \mathrm{CO}_{2}\right)$ overnight. The tissue slices were then incubated in Opti-MEM I (Invitrogen) without antioxidants and 1\% Pen-Strept and exposed to hypoxia $\left(8 \% \mathrm{O}_{2}\right)$, normoxia $\left(21 \% \mathrm{O}_{2}\right)$, or hyperoxia $\left(95 \% \mathrm{O}_{2}\right)$ for $4 \mathrm{~h}$ in the presence or absence of the ROS-sensitive fluorescent indicator, 5- (and 6)-chloromethyl-2',7'-dichlorodihydrofluorescein diacetate, acetyl ester $\left(\mathrm{CM}-\mathrm{H}_{2}\right.$ DCFDA, $2 \mu \mathrm{M}$; Molecular Probes, Eu-

Abbreviations: PG, petrosal ganglion; PG/NG, petrosal-nodose ganglion; PI, propidium iodide; ROS, reactive oxygen species 
gene, OR). Free-floating, serial sections of each PG/NG complex were exposed to each oxygen condition. Two additional experiments were performed: The first was to determine the effect of antioxidants on the tissue levels of ROS in the PG/NG complex in response to varying levels of oxygen tension. The experiment was repeated in an additional group of animals $(n=$ 6 ) at each age group and exposures were performed in the presence or absence of two antioxidants, $N$-acetyl-L-cysteine (NAC, $20 \mathrm{mM}$; Sigma Chemical Co.) and 4-[(9-acridinecarbonyl) amino]-2,2,6,6-tetramethylpiperidin-1-oxyl, free radical (TEMPO-9-AC, $10 \mu \mathrm{M}$; Molecular Probes). NAC acts both directly as a glutathione substitute and indirectly as a glutathione precursor and TEMPO9-AC is a nitroxide that acts as an antioxidant and inhibits oxidative damage by removing both extra- and intracellular $\mathrm{O}_{2}^{-{ }^{-}}$. The second was to determine the effect of hyperoxia on cell death in PG/NG neurons in the presence or absence of antioxidants. This experiment was performed in another group of animals at each age group. Propidium iodide (PI, $500 \mathrm{nM}$, Molecular Probes) was used in the presence or absence of NAC and TEMPO-9-AC during exposure to hyperoxia.

For each experiment, after exposure, sections were washed three times in PBS for $5 \mathrm{~min}$, fixed in 2\% paraformaldehyde for $10 \mathrm{~min}$, rinsed in PBS three times and for $5 \mathrm{~min}$, and then mounted on to subbed slides $(0.3 \%$ gelatin and $0.05 \%$ chromium potassium sulfate in distilled water) allowed to dry on a slide warmer for $10 \mathrm{~min}$, and cover slips were applied with ProLong Antifade Kit (Molecular Probes).

Lastly, to demonstrate morphologically which cells were being affected by hyperoxic exposure in the PG/NG complex, electron microscopy was performed on tissue taken from two animals at each age. After the tissue slices were exposed to the different oxygen tension, they were fixed in $2.5 \%$ glutaraldehyde for $10 \mathrm{~min}$ and processed for electron microscopy. Fixed $40 \mu \mathrm{m}$ sections were washed in $0.1 \mathrm{M}$ sodium cacodylate containing $3 \mathrm{mM}$ $\mathrm{CaCl}_{2}(\mathrm{pH} 7.4)$ at $4^{\circ} \mathrm{C}$. Sections were osmicated in $2 \%$ osmium tetroxide containing $0.1 \mathrm{M}$ sodium cacodylate and $3 \mathrm{mM} \mathrm{CaCl}_{2}$ for $1 \mathrm{~h}$ at $4^{\circ} \mathrm{C}$ in the dark, and then washed with deionized $\mathrm{H}_{2} \mathrm{O}$. Sections were dehydrated in an ascending series of alcohols, washed twice in propylene oxide, and infiltrated overnight in a 1:1 dilution of propylene oxide:Eponate 12 (Polysciences, Inc., Warrington, PA) containing the curing catalyst, dimethylaminoethyl phenol (DMP-30; 1.5\%). The next day, sections were embedded in Eponate 12 containing 1.5\% DMP-30 for $6 \mathrm{~h}$. Sections were flat embedded between two sheets of Aclar fluoropolymer film (Ted Pella, Inc.), weighted onto a hard flat surface and heated at $60^{\circ} \mathrm{C}$ for $2 \mathrm{~d}$. Sections were window-cut out with a razor blade and re-embedded in an inverted BEEM capsule (Ted Pella, Inc.) and cured. Blocks were trimmed on a Leica/Reichert Ultracut E ultra microtome, faced with glass knives and sectioned $(200 \mathrm{~nm})$ with a low-angle diamond knife (DiATOME, Hatfield, PA). Ultrathin tissue sections were picked up with $2 \times 1 \mathrm{~mm}$ Fomvar-coated slot grids (Ted Pella, Inc.) and photographed on a Phillips CM 120 transmission electron microscope operating at $80 \mathrm{kV}$. Negatives were converted in to tiff images with an Epson Perfection 2450 Photo Scanner operating at 400 dpi.

Data analysis. The presence of fluorescence in cell bodies in the PG/NG complex identified ROS via CM- $\mathrm{H}_{2}$ DCFDA and cell death via PI. The fluorescent cells were detected using filters for FITC (abs/em 494/519). Fluorescent images of the entire PG/NG complex from each animal were captured with a CCD camera (Photometrics CoolSnap FX; Photometrics, Tucson, AZ) attached to a fluorescent microscope (Nikon Eclipse E-400; Nikon, Tokyo, Japan) and stored in IPLab version 3.5 image analysis program. After subtracting for background fluorescence and normalization, gray level intensities for 200 ganglion cells in the PG/NG complex were measured, for each animal and each oxygen exposure. A mean gray level intensity in artificial units (a.u.) was determined for each animal, for each oxygen exposure, in each of the two age groups. Differences were determined by one-way ANOVA with posthoc analysis using the SPSS statistical program (SPSS Inc., Chicago, IL). Significance was set at $p \leq 0.05$ for post hoc analysis.

\section{RESULTS}

After $24 \mathrm{~h}$ in slice culture, the carotid body and PG/NG complexes showed no evidence of tissue disruption as observed with Hoffman-contrast microscopy (Fig. 1). Increasing $\mathrm{O}_{2}$ tension augmented the level of ROS in PG/NG neurons in tissues from both age groups, as shown qualitatively for two representative animals at DOL 5 (Fig. 2, $A-C$ ) and DOL 17 (Fig. 2, $D-F$ ) and quantitatively for all animals in each age group in Figure 3, $A$ and $B$. The mean ROS level for DOL 5 PG/NG neurons was $182.5 \pm 2.9$ for hypoxia, $217.5 \pm 3.3$ for

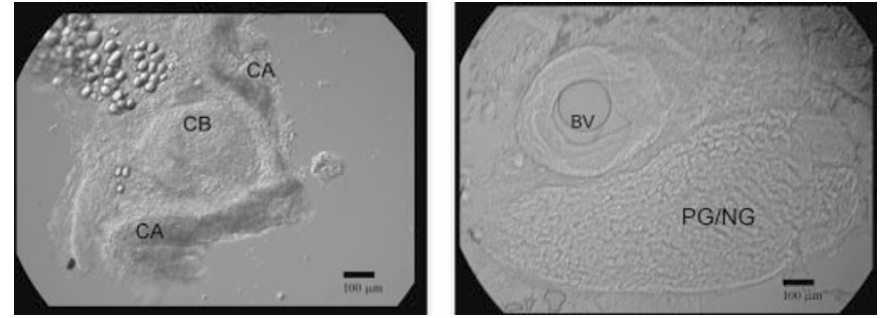

Figure 1. Low-power photomicrograph using Hoffman contrast microscopy of an organotypic slice of PG/NG after $24 \mathrm{~h}$ in culture. Sections show intact morphology with round cell bodies, visible nuclei, and without gross signs of tissue necrosis. $C B$, carotid body; $C A$, carotid artery; $B V$, blood vessel; $P G / N G$, petrosal/nodose ganglion.
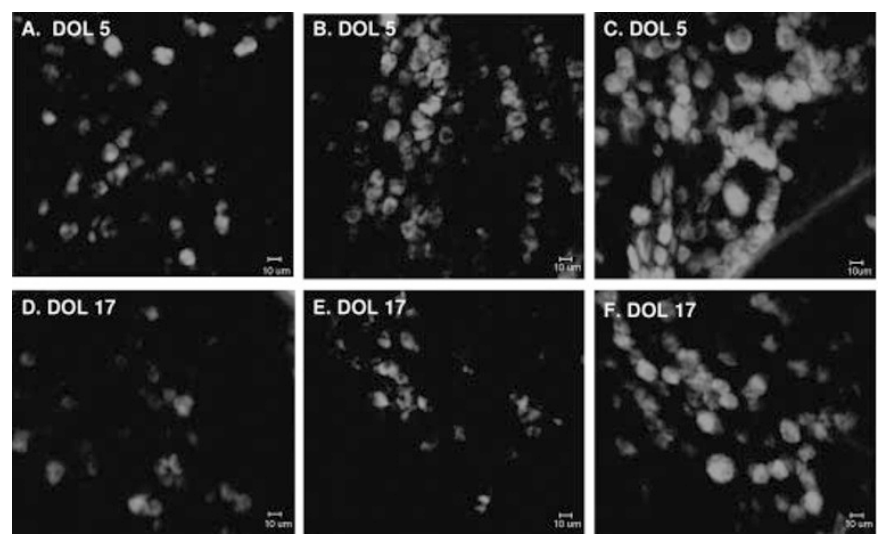

Figure 2. High-power, fluorescent photomicrograph of the PG/NG complex showing ROS levels. PG/NG complexes were taken from rat pups at DOL 5 $(A-C)$ and DOL $17(D-F)$. The ROS level is detected at each oxygen tension exposure; $8 \% \mathrm{O}_{2}(A, D), 21 \% \mathrm{O}_{2}(B, E)$, and $95 \% \mathrm{O}_{2}(C, F)$.

normoxia, and $336.6 \pm 3.8$ for hyperoxia $(p<0.001$, ANOVA; Fig. 3A). A similar increase was seen in PG/NG neurons from animals at DOL 17 with ROS levels of $217.6 \pm$ 2.9 for hypoxia, $235.5 \pm 4.3$ for normoxia, and $309.4 \pm 4.3$ for hyperoxia, $(p<0.001$, ANOVA; Fig. 3B). However, the magnitude of increased ROS level in response to increasing $\mathrm{O}_{2}$ tension differed between the two age groups. As demonstrated in Figure 4, in the younger animals, the greatest increase in ROS level was $84.7 \pm 2.0 \%$ from hypoxia to hyperoxia $(p<0.001$, ANOVA). Normoxic exposure increased ROS levels in PG/NG neurons by $19.2 \pm 1.2 \%$ in
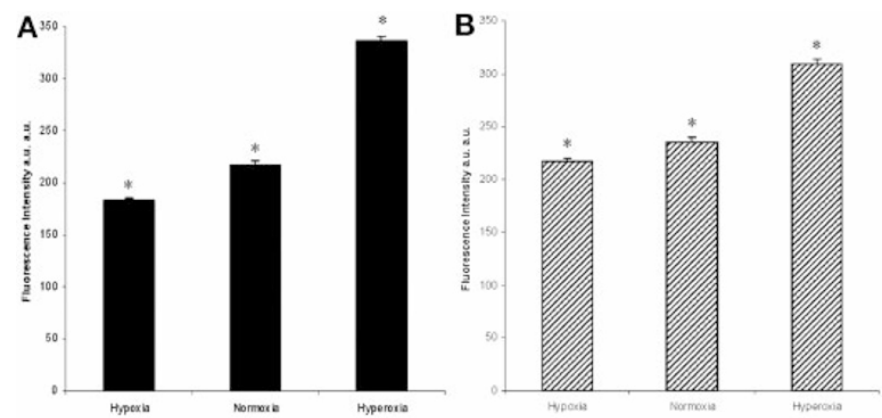

Figure 3. Mean ROS levels in response to varying $\mathrm{O}_{2}$ tension. Bar graph depicts fluorescence intensity in $\mathrm{PG} / \mathrm{NG}$ neurons in response to oxygen tension for $(A)$ DOL 5-6 $(n=10)$ and $(B)$ DOL $17-18(n=9)$. Gray-level intensities increased with increasing levels of oxygen tension, with the greatest increase noted in the $\mathrm{PG} / \mathrm{NG}$ cell bodies that were exposed to hyperoxic conditions. $p<0.001$. 


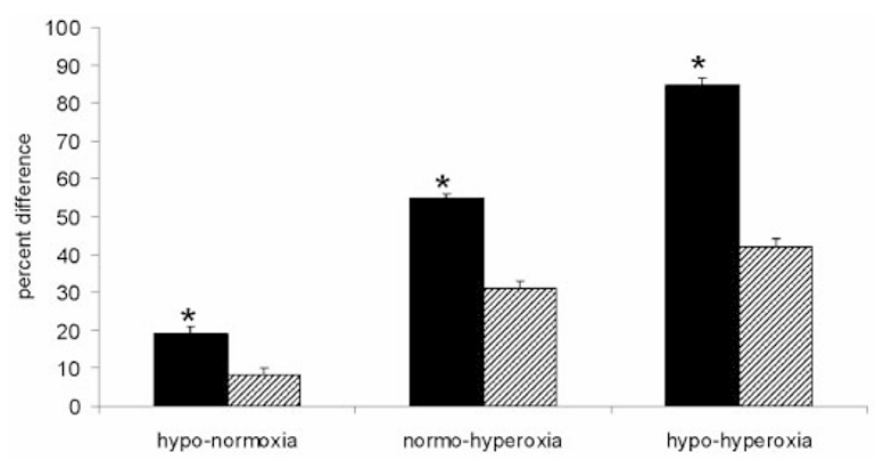

Figure 4. Percentage differences for ROS levels in response to $\mathrm{O}_{2}$ tension. Bar graph depicting developmental increases in relative percentage difference in gray-level intensity as a function of changes in oxygen tension. The percentage differences were greater for the younger animals (solid bars, $n=$ 10) than the older animals (striped bars, $n=9$ ), with the greatest relative difference demonstrated in the hypo-hyperoxia comparison. $p<0.001$.

comparison to hypoxic exposure $(p<0.01$, ANOVA) with a further increase of $54.8 \pm 1.8 \%$ from normoxia to hyperoxia $(p<0.001$, ANOVA). In the older animals, normoxic exposure increased ROS levels in PG/NG neurons by $8.2 \pm 2.0 \%$ in comparison to hypoxic exposure ( $p<0.01$, ANOVA) with a further increase of $31.2 \pm 1.8 \%$ from normoxia to hyperoxia $(p<0.001$, ANOVA). The greatest increase in ROS level was only $42.2 \pm 2.0 \%$ from hypoxia to hyperoxia $(p<0.001$, ANOVA) in the older age group, which contrasted with the $84.7 \%$ increase from hypoxia to hyperoxia, which was observed in the younger animals.

In response to hyperoxia, in the presence of the antioxidants NAC and TEMPO-9-AC, gray-level intensity representing ROS levels decreased by $18 \% \pm 3.3$ when compared with control levels, in tissues removed from animals at DOL 5-6. In contrast, in the presence of these antioxidants, ROS levels decreased by only $10.9 \pm 2.2 \%$ in the tissues of animals at DOL 17-18 versus DOL 5-6 (Fig. 5; $p<0.001$ ).

The relationship between the increase in tissue levels of ROS and cell death was determined by the number of cells that were positive for PI fluorescence as a function of oxygen tension. PI, a highly polar fluorescent molecule, penetrates damaged plasma membranes and binds irreversibly to DNA. NAC and TEMPO-9-AC attenuated the increase in levels of

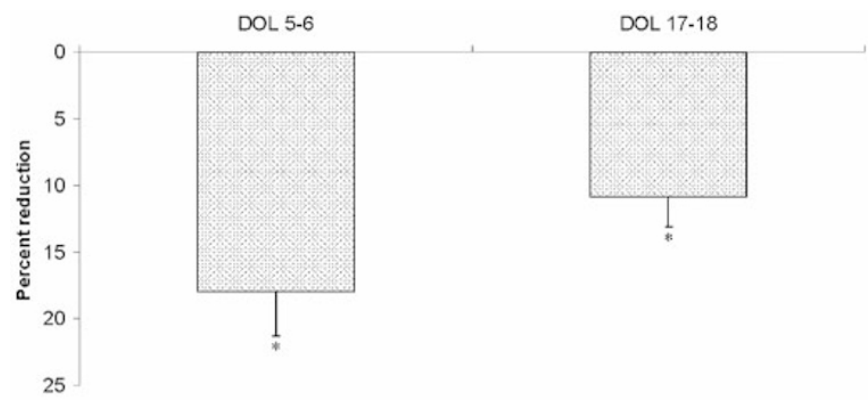

Figure 5. Attenuation of ROS levels in the presence of NAC and TEMPO9-AC. Inverted bar graph depicting a decrease in ROS levels in cell bodies in the PG in the presence of $20 \mathrm{mM}$ NAC and $10 \mu \mathrm{M}$ TEMPO-9-AC, when exposed to hyperoxic conditions. Younger animals $(n=4)$ had a greater percentage reduction in ROS levels than the older animals $(n=4) . p<0.001$, Bonferroni correction.
ROS and the number of PI positive PG/NG cells in response to increasing oxygen taken from rat pups at DOL 5-6 and 17-18, shown qualitatively for a representative animal at DOL 5 (Fig. 6) and 17 (Fig. 7) and quantitatively for all animals in Figure 8. For rats at DOL 5-6, the gray-level intensity, representing damaged cells, of the PG/NG neurons was $169.2 \pm 1.2$ for hypoxia, $185.6 \pm 1.3$ for normoxia, $289.9 \pm 1.3$ for hyperoxia, and $220.2 \pm 1.3$ for hyperoxia in the presence of NAC and TEMPO-9-AC ( $p<0.05$, ANOVA). A similar pattern of cell death was also demonstrated for animals at DOL 17-18. The gray-level intensity, representing damaged cells, of the PG/NG neurons was $149.7 \pm 1.7$ for hypoxia, $165.6 \pm 1.8$ for normoxia, $247.6 \pm 2.1$ for hyperoxia, and $205.6 \pm 1.8$ for hyperoxia in the presence of NAC and TEMPO-9-AC $(p<0.001$, ANOVA). However, when compared with the younger animals, the older animals had a comparative decrease in the percent difference of cell death in response to increasing oxygen tension from normoxia to hyperoxia ( $p<0.001$, Bonferroni correction) and from hypoxia to hyperoxia ( $p<0.001$, Bonferroni correction).

Electron microscopy demonstrated that the ganglion cells in the PG/NG complex had extensive signs of cell damage to the ultrastructure, as shown in the electron photomicrograph of a representative tissue slice taken from one animal at DOL 5 after exposure to normoxia and hyperoxia, in Figure 9, $A$ and $B$, respectively. A graded pattern of damage throughout the tissue slice was seen with some cells showing mitochondrial swelling, condensation of chromatin with minimal vacuolization of the cytoplasm while other cells showed extensive vacuolization and necrosis. Ganglion cells were also damaged in tissue sections exposed to hyperoxia from older animals, data not shown.

\section{DISCUSSION}

Using the novel technique of organotypic slices of the PG/NG complex, our data are the first to show a direct
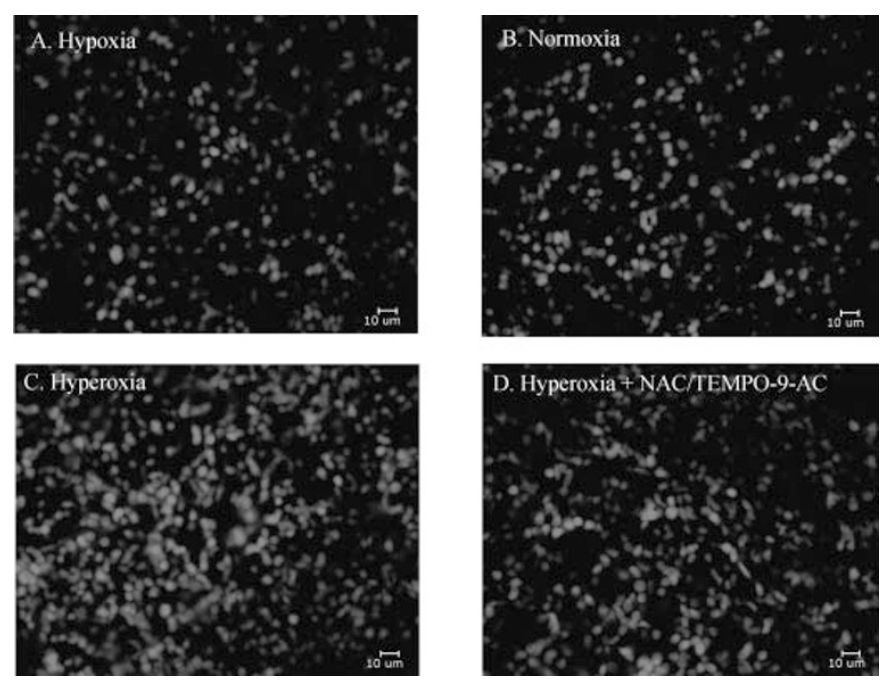

Figure 6. High-power fluorescent photomicrograph of PI-stained PG/NG neurons taken from a rat at DOL $5(A-D)$. Cell death is shown for each oxygen tension exposure $8 \% \mathrm{O}_{2}(A), 21 \% \mathrm{O}_{2}(B), 98 \% \mathrm{O}_{2}(C)$, and $98 \% \mathrm{O}_{2}$ + NAC/TEMPO-9-AC $(D)$. Fluorescent intensity is depicted in each image as brightly high-lighted cell bodies. 

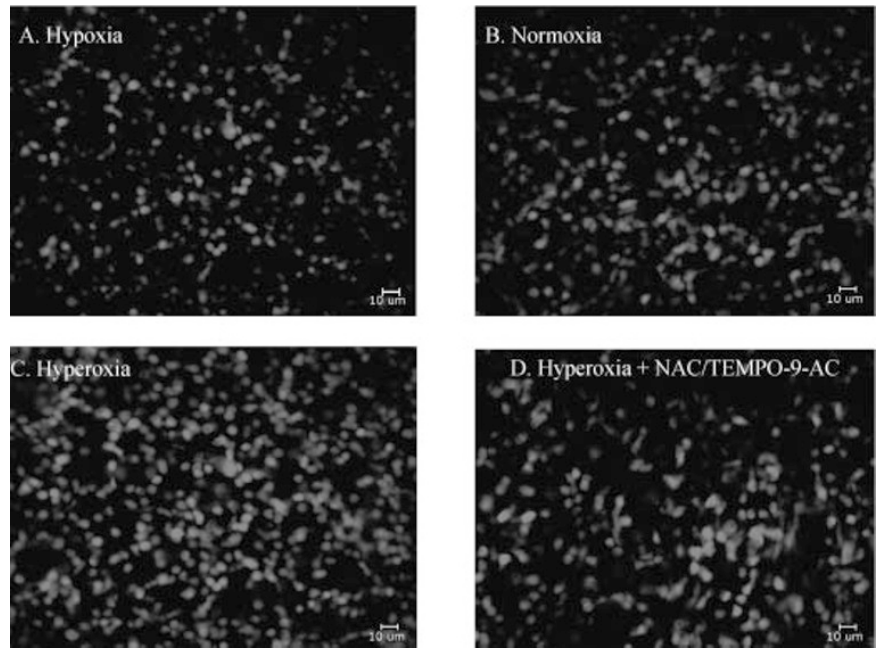

Figure 7. High-power fluorescent photomicrograph of PI-stained PG/NG neurons taken from a rat at DOL $17(A-D)$. Cell death is shown for each oxygen tension exposure $8 \% \mathrm{O}_{2}(A), 21 \% \mathrm{O}_{2}(B), 98 \% \mathrm{O}_{2}(C)$, and $98 \% \mathrm{O}_{2}$ + NAC/TEMPO-9-AC $(D)$. Fluorescent intensity is depicted in each image as brightly high-lighted cell bodies.

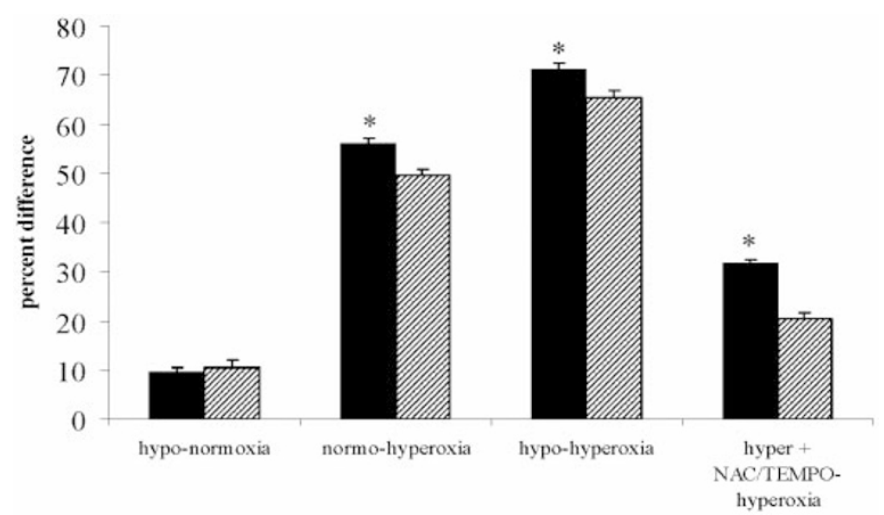

Figure 8. Percentage differences for cell death using PI in response to $\mathrm{O}_{2}$ tension. Bar graph depicting developmental increases in relative percentage difference in gray-level intensity as a function of changes in oxygen tension. The percentage differences were greater for the younger animals (solid bars, $n=10$ ) than the older animals (striped bars, $n=9$ ) except for hypoxia to normoxia, with the greatest relative difference demonstrated in the hypohyperoxia comparison. $p<0.001$, Bonferroni correction.

correlation between the level of $\mathrm{O}_{2}$ tension exposure and ROS production in PG/NG neurons. Furthermore, we show that tissues removed from rat pups within the first week of life have a greater level of ROS in PG/NG neurons in response to hyperoxia than do older rat pups. Additionally, ROS levels were attenuated by the antioxidants, NAC and TEMPO-9-AC, to a greater extent in the younger animals versus that of older animals. Cell death, as measured by PI fluorescence, increased with exposure to increasing $\mathrm{O}_{2}$ tension in the younger animals. Although the older animals also had an increase in cell death with increasing oxygen tension, they had comparatively less cell death than the younger animals at the higher oxygen tensions. Our electron microscopy studies demonstrate that increasing oxygen tension damages cells that are the soma of the ganglion cells in the PG/NG complex. Some of these soma are most likely from chemoafferent cells. Increased tissue

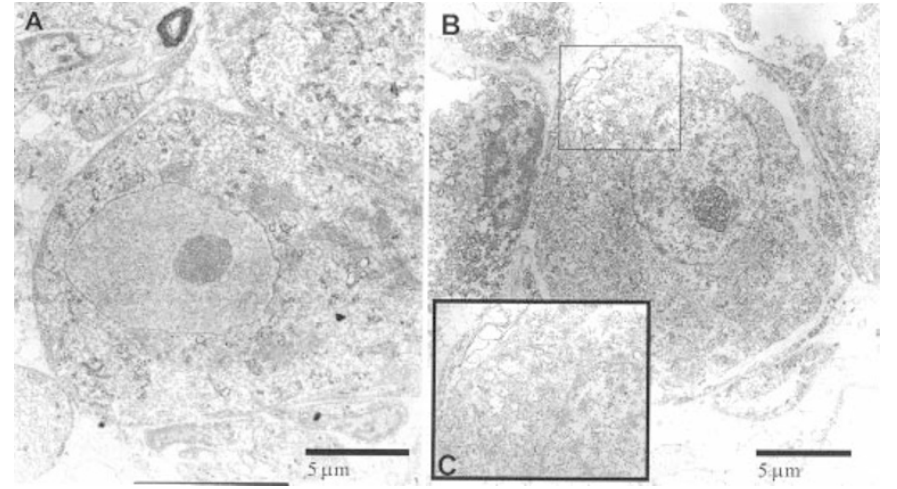

Figure 9. Electron micrograph of petrosal ganglion cells after exposure to normoxia $(A)$ and hyperoxia $(B)$. Ganglion cells exposed to normoxia have intact nuclear and cellular membranes, and normal-appearing chromatin and nucleoli. Prominent ribosomes are seen throughout. Ganglion cells exposed to hyperoxia demonstrate severe injury, including condensation of chromatin along the nuclear membrane, rarefaction, and vacuolization of the cytoplasm. No intact mitochondria are found in the hyperoxia exposed cell.

levels of ROS within chemoafferent cell bodies may account for histologic evidence of cytotoxicity and ablation of hypoxic chemosensitivity in these cells, in newborn rats exposed to hyperoxia (10).

Dissociated cell cultures of lung and brain are used to examine the effects of varying $\mathrm{O}_{2}$ tension levels on ROS production and cell damage (for review, see Ref. 11). ROS production in nodose ganglia during acute hypoxia in $100-\mu \mathrm{m}$ slice cultures from adult rats has been described (12). In the present study, we used $45-\mu \mathrm{m}$ organotypic slices of the PG/NG from newborn rats to ascertain the effect of varying oxygen levels on tissue levels of ROS. Our technique has several advantages over assays using dissociated cells in culture and thicker organotypic slices, which include maintaining the ultrastructure of the peripheral arterial chemoreceptor complex while still allowing for uniform diffusion of $\mathrm{O}_{2}$ and reagents to the target cells. Using thinner organotypic slices, we are able to further delineate the effects of hyperoxia on the PG/NG complex in a neonatal rat model that corroborates previous histologic findings described by Erickson et al. (8) in the rat carotid body and nerve fibers and cell bodies of chemoafferents.

ROS, e.g. superoxide and hydroxy radicals, are constantly generated as byproducts of cellular oxidative metabolism, but their production is increased in various pathologic states, and also upon exposure to exogenous oxidants, such as hyperoxia (13). The short half-life and the high reactivity of ROS limit the direct detection of these compounds in vivo. As radicals have an unpaired electron, direct detection of radicals is possible by electron spin resonance (ESR), but the concentrations of radicals reached in living tissue are below the detection limit of this method. Fluorometric and luminescencebased assays, thus, are used to measure these radicals in tissues and cells. Fluorometric techniques, different to luminescence measurements, allow the topolocalization of ROS in relatively small tissues and at small production levels (14). We used a derivative of reduced fluorescein and calcein as cellpermeant indicator for ROS. Chemically reduced and acety- 
lated forms of 2',7'-dichlorofluorescein (DCF) and calcein are nonfluorescent until the acetate groups are removed by intracellular esterases and oxidation occurs within the cell. Esterase cleavage of the lipophilic blocking groups yields a charged form of the dye that is much better retained by cells than is the parent compound. Oxidation of these probes are then detected by monitoring the increased fluorescence with a fluorescence microscope, using excitation sources and filters appropriate for fluorescein (FITC).

Increased ROS levels may lead to cell death accounting for the reduction in the number of chemoafferents in newborn animals exposed to hyperoxia. Our data, using PI, corroborates Erickson et al. (8) findings that there was a decrease in the number of chemoafferents after exposure to hyperoxia. PI is a fluorescent dye that is membrane impermeant and generally excluded from viable cells. It binds to DNA by intercalating between the bases with little or no sequence preference and once bound to nucleic acids, its fluorescence is enhanced 20 - to 30-fold. PI is commonly used for identifying dead cells in a population, and in this study, it was used to determine the relationship between cell death and levels of ROS in PG neurons and varying oxygen tension in both the younger and older animals. PI fluorescence was increased with increasing oxygen tension, indicating that not only does hyperoxia increase levels of ROS, but that there is histologic evidence of damage to the cells. Additionally, our data suggest a developmental response to oxidative stress with older animals having less cell death than do younger animals for all of the conditions that were tested. The presence of NAC and TEMPO-9-AC attenuated this response, which also suggests an association between increased ROS levels and cell death.

Antioxidants attenuated the level of ROS production in response to hyperoxia. Both NAC and TEMPO-9-AC have antioxidant activities that may contribute to protection against oxidative stress. NAC scavenges free radicals and augments intracellular levels of glutathione (15). TEMPO-AC-9 is a nitroxide that acts as an antioxidant detoxifying ferryl heme species, facilitating heme-mediated catalytic removal of $\mathrm{H}_{2} \mathrm{O}_{2}$, trapping carbon-centered radicals and terminating radical chain reactions. However, the most important mechanism is that nitroxides inhibit oxidative damage by removing both extra- and intracellular $\mathrm{O}_{2}{ }^{--}$(16). Our findings demonstrate antioxidants are more effective in preterm versus term rat pups. This may be due to reduced production of antioxidants, increased production of oxidative species, or a decrease in antioxidant activity (17). Our data do not allow use to speculate how a particular antioxidant may affect hyperoxic damage in the peripheral arterial chemoreceptors in human infants. Of interest, the use of NAC in preterm infants during the first week of life has not been shown to reduce the incidence of bronchopulmonary dysplasia $(18,19)$, another morbidity of premature birth that is associated with hyperoxic exposure. Preterm infants may not effectively deacetylate NAC to cysteine, which is necessary for the molecule to function as a precursor for glutathione (20).

Perinatal hyperoxic exposure severely depresses hypoxic chemosensitivity, as recorded from carotid sinus nerves of kittens (21) and adult rats (10), suggesting impairment of carotid body chemoreflex. Prolonged exposure of newborn rats to $60 \% \mathrm{O}_{2}$ results in marked chemoafferent neuron degeneration as well as carotid body hypoplasia and blunting of the hypoxic chemoreflexes $(6,8)$. Several potential mechanisms have been proposed by which chronic hyperoxia leads to ablation of hypoxic chemosensitivity, carotid body hypoplasia, and a reduced number of chemoafferents. These models suggest that prolonged perinatal hyperoxia could decrease the size of the carotid body by directly attenuating cell proliferation, or inducing cell death. Whereas it is known that the carotid body has trophic influences on chemoafferent neurons $(22,23)$, hyperoxia may not be selective in its effects and could have a direct influence on chemoafferent neurons within the PG/NG complex itself. Our data suggest that PG/NG neurons taken from neonatal rats and exposed to hyperoxia may respond similarly to previous findings by Erickson et al. (8) in the rat carotid body in response to hyperoxia and that even at normoxic levels of oxygen tension increased cell death and higher levels of ROS occur.

We have interpreted our data being aware of the following potential limitations of the technique and method. Due to the small size of the tissues in the younger animals, we chose not to try to separate the nodose from petrosal ganglia before embedding the tissue in the agar blocks to achieve good morphology with little tissue disruption. Thus, we show that hyperoxia affects ROS levels in both nodose and petrosal ganglion neurons. The increase in tissue levels of ROS, using the fluorometric technique, does not allow us to determine whether there is an increase in production versus a decrease in antioxidant. Lastly, because the data from Erickson et al. demonstrated that there was a $41 \%$ reduction in chemoafferents in response to hyperoxic exposure, we chose to determine whether there was a direct effect of hyperoxia on ROS levels in chemoafferents and did not systematically assay for changes in ROS levels in the carotid body.

In conclusion, using a novel technique of organotypic slices of the PG/NG, our data show a direct correlation between the level of oxygen tension exposure to cell bodies of chemoafferent neurons and ROS levels. Increased ROS levels within chemoafferent cell bodies may account for histologic evidence of cytotoxicity and ablation of hypoxic chemosensitivity in newborn animals that is life-long. Our data may be relevant to disorders of respiratory control that have been described in premature infants. Premature infants with chronic lung disease have blunted chemoreceptor responses (7). In the preterm neonate, oxygen-based postnatal therapy is necessary to maintain levels of arterial $\mathrm{P}_{2}$ within normal limits. However, the premature infant has reduced defense capabilities to free radical damage, thereby resulting in oxidative injury to developing tissues (17). Thus, the preterm neonate is more vulnerable to disorders of antioxidant-prooxidant balance and disorders of physiologic function as a result of cellular and tissue injury from free radicals, including the persistence of the arterial ductus, persistent pulmonary hypertension, necrotizing enterocolitis, retinopathy of prematurity, chronic lung disease, hypoxic-ischemic encephalopathy, and intraventricular hemorrhage. We speculate that blunted chemoreceptor responses in newborn infants with chronic lung disease may be related to 
hyperoxia-induced cytotoxicity of key cells and neurons in the peripheral arterial chemoreceptors and that normoxic exposure of the premature infant may potentially induce cytotoxic effects upon these same cells and neurons.

Acknowledgments. The authors thank Gabrielle L. McLemore, Ph.D., for her assistance in editing the manuscript; Reed Z. Cooper, M.S., and Ariel V. Mason for their technical support; and Frances J. Northington, M.D., for her assistance with the interpretation of the electron micrographs.

\section{REFERENCES}

1. Gauda EB, McLemore GL, Tolosa J, Marston-Nelson J, Kwak D 2004 Maturation of peripheral arterial chemoreceptors in relation to neonatal apnoea. Semin Neonatol 9:181-194

2. Carroll JL 2003 Developmental plasticity in respiratory control. J Appl Physiol 94:375-389

3. Gonzalez C, Almaraz L, Obeso A, Rigual R 1994 Carotid body chemoreceptors: from natural stimuli to sensory discharges. Physiol Rev 74:829-898

4. Verna A 1997 The mammalian carotid body: morphological data. In: Gonzalez C (ed) The Carotid Body Chemoreceptors. Springer, New York, pp 1-29

5. Ling L, Olson EB Jr, Vidruk EH, Mitchell GS 1997 Integrated phrenic responses to carotid afferent stimulation in adult rats following perinatal hyperoxia. J Physiol 500:787-796

6. Ling L, Olson EB Jr, Vidruk EH, Mitchell GS 1997 Phrenic responses to isocapnic hypoxia in adult rats following perinatal hyperoxia. Respir Physiol 109:107-116

7. Katz-Salamon M, Jonsson B, Lagercrantz H 1995 Blunted peripheral chemoreceptor response to hyperoxia in a group of infants with bronchopulmonary dysplasia. Pediatr Pulmonol 20:101-106

8. Erickson JT, Mayer C, Jawa A, Ling L, Olson EB Jr., Vidruk EH, Mitchell GS, Katz DM 1998 Chemoafferent degeneration and carotid body hypoplasia following chronic hyperoxia in newborn rats. J Physiol 509:519-526

9. Jamieson D, Chance B, Cadenas E, Boveris A 1986 The relation of free radical production to hyperoxia. Annu Rev Physiol 48:703-719

10. Bisgard GE, Olson EB Jr., Wang Z-Y, Bavis RW, Fuller DD, Mitchell GS 2003 Adult carotid chemoafferent responses to hypoxia after 1,2, and 4 wk of postnatal hyperoxia. J Appl Physiol 95:946-952
11. Asikainen TM, White CW 2004 Pulmonary antioxidant defenses in the preterm newborn with respiratory distress and bronchopulmonary dysplasia in evolution: implications for antioxidant therapy. Antioxid Redox Signal 6:155-167

12. Yamamoto Y, Henrich M, Snipes RL, Kummer W 2003 Altered production of nitric oxide and reactive oxygen species in rat nodose ganglion neurons during acute hypoxia. Brain Res 961:1-9

13. Halliwell B, Gutteridge JM 1989 Free Radicals in Biology and Medicine, 2nd Ed Clarendon Press, Oxford, pp 45-50

14. Tarpey MM, Fridovich I 2001 Methods of detection of vascular reactive species: nitric oxide, superoxide, hydrogen peroxide, and peroxynitrite. Circ Res 89:224-236

15. Kyaw M, Yoshizumi M, Tsuchiya K, Izawa Y, Kanematsu Y, Fujita Y, Ali N, Ishizawa K, Yamauchi A, Tamaki T 2004 Antioxidant effects of stereoisomers of $\mathrm{N}$-acetylcysteine (NAC), L-NAC and D-NAC, on angiotensin II-stimulated MAP kinase activation and vascular smooth muscle cell proliferation. J Pharmacol Sci 95:483-486

16. Offer T, Mohsen M, Samuni A 1998 An SOD-mimicry mechanism underlies the role of nitroxides in protecting papain from oxidative inactivation. Free Radic Biol Med 25:832-838

17. Brady R, Zaidi SIA, Mayer C, Katz DM 1999 BDNF is a target-derived survival factor for arterial baroreceptor and chemoafferent primary sensory neurons. J Neurosci 19:2131-2142

18. Sandberg K, Fellman V, Stigson L, Thiringer K, Hjalmarson O $2004 \mathrm{~N}$ Acetylcysteine administration during the first week of life does not improve lung function in extremely low birth weight infants. Biol Neonate 86:275-279

19. Ahola T, Lapatto R, Raivio KO, Selander B, Stigson L, Jonsson B, Jonsbo F, Esberg G, Stovring S, Kjartansson S, Stiris T, Lossius K, Virkola K, Fellman V 2003 $\mathrm{N}$-Acetylcysteine does not prevent bronchopulmonary dysplasia in immature infants: a randomized controlled trial. J Pediatr 143:713-719

20. Van Goudoever JB, Sulkers EJ, Timmerman M, Huijmans GM, Langer K, Carniellu VP, Sauer D 1994 Amino acid solutions for premature neonates during the first week of life: the role of $N$-acetyl-L-cysteine and $N$-acetyl-L-tyrosine. JPEN J Parenter Enteral Nutr 18:404-408

21. Hanson MA, Eden GJ, Nijhuis JG, Moore PJ 1989 Peripheral chemoreceptors and other oxygen sensors in the fetus and newborn. In: Lahiri S, Forster RE, Davies RO, Pack AI (eds) Chemoreceptors and Reflexes in Breathing: Cellular and Molecular Aspects. Oxford University Press, New York, pp 113-120

22. Hertzberg T, Fan G, Finley JCW, Erickson JT, Katz DM 1994 BDNF supports mammalian chemoafferent neurons in vitro and following peripheral target removal in vivo. Dev Biol 16:801-811

23. Ling L, Olson EB Jr, Vidruk EH, Mitchell GS 1996 Attenuation of the hypoxic ventilatory response in adult rats following one month of perinatal hyperoxia J Physiol 495:561-565 